\title{
Análisis de antenas lineales por el método de momentos
}

\author{
Rodolfo Neri-Vela \\ División de Ingeniería Eléctrica, Facultad de Ingeniería, UNAM \\ Email: aldeca@lcomp98.fi-b.unam.mx
}

(recibido: octubre de 1998; aceptado: diciembre de 1998)

\begin{abstract}
Resumen
El método de momentos se ha convertido en una herramienta muy útil para analizar las propiedades de operación de un gran número de antenas empleadas en radiocomunicaciones. Este artículo ofrece un procedimiento matemático sencillo y preciso de dicho método, el cual se ejemplifica con algunos resultados obtenidos para una antena Yagi-Uda. El procedimiento puede servir como apoyo didáctico en los cursos de ingeniería.
\end{abstract}

\section{Abstract}

The moment method of analysis in antenna theory has become a very useful tool to study the operation properties of a large number of radiating structures. In this paper, a precise and easy procedure explaining the method is given, together with some numerical results obtained for a Yagi-Uda. The approach given can help students and teachers on the subject to better understand the method, specially if they are facing it for the first time.

\section{Introducción}

Hace un par de décadas aún era difícil emplear el método de momentos (Harrington, 1968) para analizar las propiedades de radiación de la mayor parte de las antenas lineales, especialmente si éstas tenían una longitud eléctrica total muy larga, de muchas longitudes de onda. Las computadoras personales y los procesadores modernos han modificado esta situación radicalmente. El principal problema del pasado, que consistía en la falta de capacidad de memoria y, particularmente, en la necesidad de invertir las matrices complejas involucradas, muchas veces por el método de partición de matrices, ya ha sido resuelto. Ahora, la mayor parte de los libros de texto sobre antenas más recientes mencionan al método de momentos como una herramienta muy útil para estudiar una gran variedad de estructuras radiantes. El tema resulta obligado en cualquier curso de licenciatura o de posgrado en las instituciones que forman a los nuevos ingenieros en telecomunicaciones o en comunicaciones y electrónica, pues no sólo brinda al alumno la oportunidad de practicar sus habilidades de programación por computadora e impresión láser, sino que éste adquiere un conocimiento más sólido sobre las propiedades de las antenas y cómo cambian las mismas al variar alguno de los parámetros en su programa.
Sin embargo, a pesar de la actualidad e importancia, tanto académica como práctica (Tsai, 1978) del método de momentos, no existe una referencia sencilla que permita a los profesores de la materia introducir dicho tema ante sus alumnos, y aun menos en nuestro idioma. El objetivo de este artículo es ofrecer dicha herramienta didáctica que falta en nuestra literatura, de modo que sea útil tanto para la docencia como para el fabricante y el profesional. El procedimiento aquí expuesto emplea funciones escalón para aproximar o discretizar la distribución de las corrientes en los conductores. Desde luego, es posible utilizar otro tipo de funciones, como por ejemplo, corrientes triangulares superpuestas, pero la distribución escalonada es la más sencilla y proporciona resultados muy exactos si se usa el número suficiente de segmentos, y esto ya no es ningún obstáculo para las computadoras de nues. tros días. Aunque el enfoque de este artículo es hacia el vasto campo de las antenas lineales, es pertinente mencionar que el método también se utiliza extensamente para el análisis y diseño de arreglos con control de fase, discontinuidades en guías de ondas, y muchos otros problemas sobre antenas (Wang, 1990).

\section{Descripción general del método}

La aplicación del método de momentos a un conductor de forma arbitraria consiste, inicialmente, en dividirlo en un 
número $N$ determinado de segmentos, mismos que pueden o no tener la misma longitud. Cada segmento tiene una impedancia propia asociada; además, debido a la proximidad física entre todos los segmentos del conductor, también existe una impedancia mutua entre cada par de ellos. La relación entre los voltajes y las corrientes de los segmentos, con las impedancias propias y mutuas, se puede expresar como

$$
\begin{aligned}
& V_{1}=I_{1} Z_{11}+I_{2} Z_{12}+\ldots+I_{N} Z_{1 N} \\
& V_{2}=I_{1} Z_{21}+I_{2} Z_{22}+\ldots+I_{N} Z_{2 N} \\
& \vdots \\
& V_{N}=I_{1} Z_{N 1}+I_{2} Z_{N 2}+\ldots+I_{N} Z_{N N}
\end{aligned}
$$

Donde $V_{i}$ es la diferencia de voltaje asociada con el segmento $i, I_{i}$ es la corriente que fluye en él, $Z_{i i}$ es su impedancia propia y $Z_{i j}$ es la impedancia mutua entre los segmentos " $i$ " y " $j$ ".

Cuando se tiene una antena con dos o más conductores, también se puede escribir un sistema de ecuaciones simultáneas semejante al anterior.

El sistema de ecuaciones 1 se puede representar en forma matricial:

$$
[V]=[Z][I]
$$

donde [V] e [I] son arreglos de $N$ elementos y [Z] es una matriz de impedancias, cuadrada y de $\operatorname{orden} N$. Si [V] y [Z] se conocen, es posible encontrar la distribución de corrientes [I], y a partir de ella, las características de radiación de la antena.

De la ecuación 2 se tiene que

$$
[I]=[Z]^{-1}[V]
$$

Estos conceptos pueden aclararse mediante la figura 1, donde se muestra una sección de un conductor de forma arbitraria. A lo largo de ella se indican dos segmentos, denotados por " $m$ " y " $n$ "; es claro que sus longitudes no son necesariamente iguales.

Debido a la presencia de una corriente y de cargas eléctricas en el segmento $n$, se produce un campo eléctrico en el segmento $m$, que induce una diferencia de potencial entre los extremos del mismo. La dependencia de este campo eléc. trico con respecto a la corriente del segmento $n$ y la distribución de las cargas asociadas con ella, se puede expresar en función del potencial magnético $\vec{A}$ que produce la primera, y del potencial eléctrico $V$ producido por las segundas. Para el punto específico $\hat{m}$ de la figura, esta relación resulta ser, de acuerdo con las ecuaciones de Maxwell:

$$
\vec{E}^{i}(\hat{m})=-j \omega \vec{A}(\hat{m})-\vec{\nabla} V(\hat{m})
$$

Si la longitud del segmento $m$ es suficientemente pequeña, se puede considerar que el valor promedio de la intensidad del campo eléctrico producido en cualquier punto de su superficie es igual a la del punto medio $\hat{m}$. Bajo esta consideración, y empleando la ley de $\mathrm{Ohm}$, la impedancia mutua entre los segmentos $m$ y $n$ se puede evaluar como

$$
Z_{m n}=\frac{-\vec{E}^{i}(\hat{m}) \cdot \Delta \vec{l}_{\hat{m}}}{I_{n}}
$$

De acuerdo con el conocido efecto piel, la corriente en un conductor fluye sobre o cerca de la superficie del mismo, decreciendo su magnitud exponencialmente hacia el centro del conductor. Sin embargo, existen estudios matemáticos que han demostrado la validez de suponer, bajo ciertas restricciones, que la corriente fluye exclusivamente a lo largo del eje central del conductor, es decir, que se tiene un filamento de corriente en el centro (Neri, 1978).

El producto escalar de la ecuación 5 indica que si $\Delta l_{\hat{m}}$ se aproxima a una línea recta, entonces es suficiente encontrar la componente tangencial de $\vec{E}^{i}(\hat{m})$ y efectuar un producto ordinario para encontrar la impedancia mutua $Z_{m n}$. Esto es válido siempre y cuando la longitud de cada segmento sea igual o menor a $\lambda / 10$; además, la superficie del segmento no debe tener cambios muy bruscos de orientación.

Para poder calcular $\vec{E}^{i}(\hat{m})$ con la ecuación 4 , se requiere obtener inicialmente el componente tangencial de $\vec{A}(\hat{m})$. En la figura 1, este componente forma un ángulo $\beta$ con $\vec{A}$, y puede expresarse como

$$
A_{t}(\hat{m})=\frac{\mu}{4 \pi} \int_{\Delta l_{n}} I_{n} \cos \beta \frac{e^{-j k R_{\hat{m}}}}{R_{\hat{m}}} d l
$$

Dado que la longitud de cada segmento se ve limitada a $\lambda / 10$ o menos, es posible considerar a cada uno de ellos como un dipolo corto, con corriente de magnitud y fase aproximadamente constantes. De aquí que la corriente $I_{n}$ en la ecuación 6 anterior se pueda escribir fuera del símbolo de integración, lo que conduce a aproximar la distribución de corrientes a lo largo del conductor por una distribución escalonada. Esto se ejemplifica en la figura 2, para un conductor arbitrario dividido en cinco segmentos iguales y dos semi-segmentos en los extremos. Por conveniencia, la corriente en estos semi-segmentos extremos se asume igual a cero, con base en la teoría de líneas de transmisión, ya que cada elemento o tubo de una antena lineal se puede interpretar como una línea terminada en circuito abierto. 


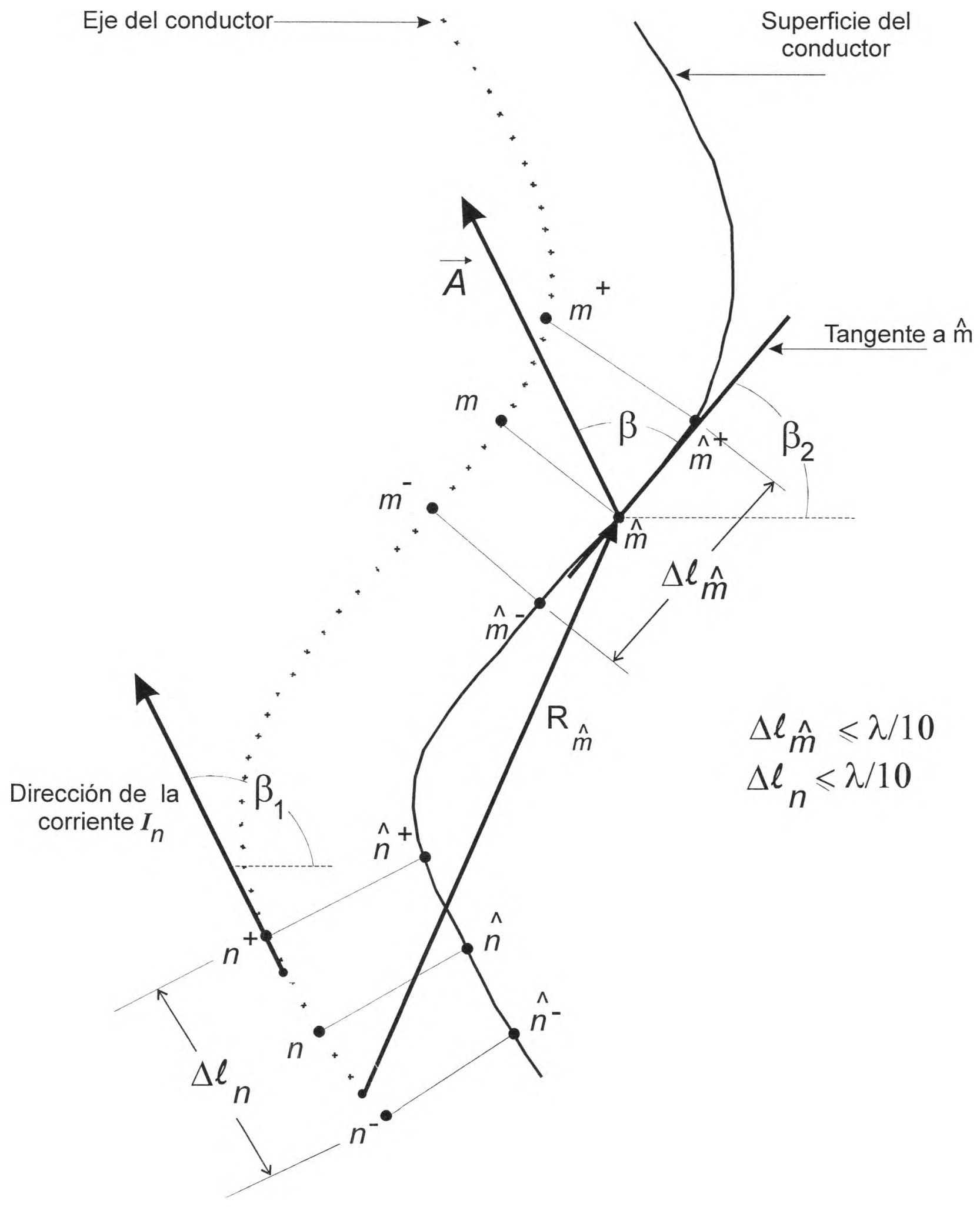

Figura 1. Sección de un conductor dividido en segmentos con longitud menor o igual que $\lambda / 10$. Se muestra la posición de dos segmentos arbitrarios $m$ y $n$ 


\section{Distribución de corriente escalonada}

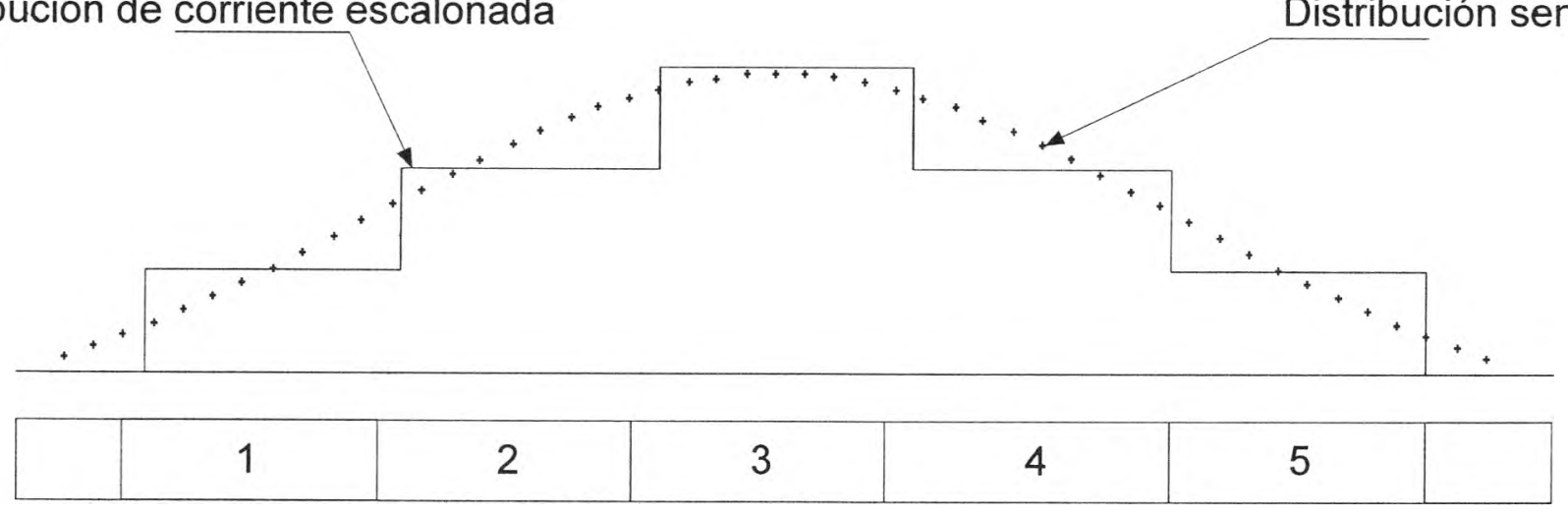

Figura 2. Conductor dividido en cinco segmentos más dos semisegmentos en los extremos y una posible distribución de corriente escalonada

Aun cuando la corriente en un segmento específico se considere constante en magnitud y fase, su dirección puede cambiar y, por lo tanto, este efecto sí debe tomarse en cuenta. De ahí que el ángulo $\beta$, que es función de la variable de línea $l$, debe permanecer en el integrado. La ecuación 6 se puede escribir entonces como

$$
A_{t}(\hat{m})=\frac{\mu}{4 \pi} I_{n} \int_{\Delta l_{n}} \cos \beta \frac{e^{-j k R_{\hat{m}}}}{R_{\hat{m}}} d l
$$

o bien:

$$
A_{t}(\hat{m})=\frac{\mu}{4 \pi} \Delta I_{n} I_{n} \psi(n, \hat{m})
$$

donde

$$
\psi(n, \hat{m})=\frac{1}{\Delta l_{n}} \int_{\Delta l_{n}} \cos \beta \frac{e^{-j k R_{\hat{m}}}}{R_{\hat{m}}} d l
$$

y $k$ es la constante de propagación en el espacio libre.

En la ecuación 4 también se requiere conocer el gradiente del potencial escalar $V$. Para esto, la experiencia ha demostrado que un modelo de distribución de cargas eléctricas como el de la figura 3 da resultados prácticos muy satisfactorios.

Para una corriente $I_{n}$ que fluye en el segmento $n$, la densidad de carga a lo largo del intervalo $[n, n+1]$ es igual a
$\left(\frac{I_{n}}{j \omega}\right)\left(\frac{1}{\Delta l_{n^{+}}}\right) ;$acordemente, la densidad de carga a lo largo del intervalo $[n-1, n]$ es igual a $\left(\frac{-I_{n}}{j \omega}\right)\left(\frac{1}{\Delta l_{n^{-}}}\right) . \Delta l_{n^{+}}$denota la longitud del intervalo que une los puntos medios de los segmentos $n$ y $n+1$, y $\Delta l_{n^{-}}$denota la longitud del intervalo limitado por los puntos medios de los segmentos $n-1$ y $n$.

El potencial escalar $V$ producido en los puntos extremos del segmento $m$ por un filamento de corriente $I_{n}$ se puede expresar en cada caso como la suma algebraica de dos integrales:

$$
\begin{aligned}
V\left(\hat{m}^{+}\right) & =\frac{1}{4 \pi \varepsilon}\left[\int_{\Delta l}\left(\frac{I_{n}}{j \omega \Delta l}\right)\left(\frac{e^{-j k R_{n^{+}}}}{R_{\hat{m}^{+}}}\right) d l\right. \\
& \left.-\int_{\Delta l_{n^{-}}}\left(\frac{I_{n}}{j \omega \Delta l_{n^{-}}}\right)\left(\frac{e^{-j k R_{\hat{m}^{+}}}}{R_{\hat{m}^{+}}}\right) d l\right]
\end{aligned}
$$

$$
\begin{aligned}
V\left(\hat{m}^{-}\right)= & \frac{1}{4 \pi \varepsilon}\left[\int_{\Delta l}\left(\frac{I_{n}}{j \omega \Delta l}\right)\left(\frac{e^{-j k R_{n^{+}}}}{R_{\hat{m}^{-}}}\right) d l\right. \\
& \left.-\int_{\Delta l}\left(\frac{I_{n}}{j \omega \Delta l_{n^{-}}}\right)\left(\frac{e^{-j k R_{\hat{m}^{-}}}}{R_{\hat{m}^{-}}}\right) d l\right]
\end{aligned}
$$




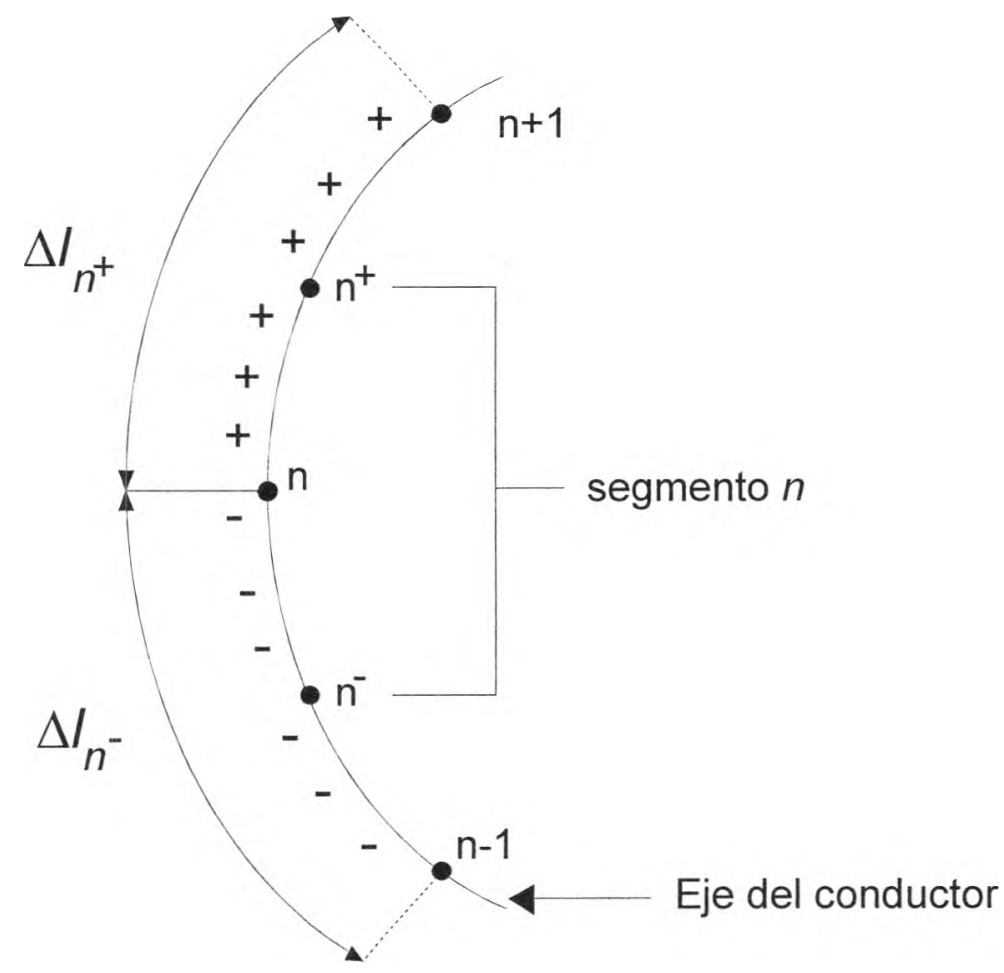

Figura 3. Modelo de distribución de las cargas eléctricas para el segmento $n$

Ahora bien, el gradiente del potencial escalar está dado por:

$$
\nabla V \approx \frac{\partial V}{\partial l}
$$

donde $l$ denota la variable de longitud a lo largo de la cual varía $V$. La derivada de la ecuación 12 se puede aproximar por una diferencia finita sobre el intervalo de diferenciación:

$$
\nabla V \approx \frac{V\left(\hat{m}^{+}\right)-V\left(\hat{m}^{-}\right)}{\Delta l_{\hat{m}}}
$$

Sustituyendo las ecuaciones 10,11 y $9 \operatorname{con} \cos \beta=1$ en la ecuación 13 , se tiene

$$
\nabla V=\frac{I_{n}}{4 \pi \varepsilon j \omega \Delta l_{\hat{m}}}
$$

$$
\left[\psi\left(n^{+}, \hat{m}^{+}\right)-\psi\left(n^{-}, \hat{m}^{+}\right)-\psi\left(n^{+}, \hat{m}^{-}\right)+\psi\left(n^{-}, \hat{m}^{-}\right)\right]
$$

donde $\psi\left(n^{+}, \hat{m}^{+}\right)$indica que el intervalo de integración tiene como centro a $n^{+}$y que todas las distancias $R$ se toman a partir de puntos sobre este intervalo hacia el punto $\hat{m}^{+}$. El resto de las funciones $\psi$ tienen una interpretación similar.

Al sustituir las ecuaciones 8 y 14 en la ecuación 4 , y utilizando la ecuación 5 , se llega a la expresión final que permite calcular las impedancias propias y mutuas:

$$
\mid \underbrace{Z_{m n}=\frac{j \omega \mu}{4 \pi} \Delta l_{n} \Delta l_{\hat{m}} \psi(n, \hat{m})+}_{\begin{array}{c}
\text { Contribución } \\
\text { de las corrientes }
\end{array}}
$$$$
\frac{1}{j 4 \pi \varepsilon \omega}\left[\psi\left(n^{+}, \hat{m}^{+}\right)-\psi\left(n^{-}, \hat{m}^{+}\right)-\psi\left(n^{+}, \hat{m}^{-}\right)+\psi\left(n^{-}, \hat{m}^{-}\right)\right]
$$ 
Es importante recordar que el factor $\cos \beta$ siempre es igual a 1 en las cuatro funciones $\psi$ del segundo término de la ecuación 15. Al aplicar esta ecuación a todas las parejas posibles de segmentos, para calcular las impedancias mutuas, y a cada segmento en particular, para obtener su impedancia propia, se forma finalmente la matriz de impedancias $[Z]$. Después de invertir dicha matriz y sustituir el resultado en la ecuación 3 , se obtiene la distribución escalonada de corrientes en el conductor (o conductores).

En el caso de que la antena sea alimentada en un solo punto, todos los elementos del arreglo de voltajes en la ecuación 3 son iguales a cero, con excepción del correspondiente al segmento donde se alimenta la antena. Por conveniencia, este voltaje de alimentación se toma igual a $1 \angle 0^{\circ}$ volts; es decir, la distribución de corrientes $[I]$ es igual a la columna $i$ de la matriz $\left[Z^{-1}\right]^{-1}$ cuando la antena se alimenta en el segmento $i$.

\section{Obtención de las propiedades de la antena}

Una vez conocida la distribución de corrientes en el conductor o conductores de la antena, el procedimiento para determinar sus propiedades radioeléctricas es relativamente simple, haciendo uso de las ecuaciones de Maxwell. A continuación se indica cómo pueden determinarse:

\section{1) Patrón de radiación}

Para graficar el patrón de radiación se requiere calcular la intensidad del campo eléctrico producido por la distribución de corrientes $[I]$ en puntos suficientes de observación $\mathrm{P}(x, y, z)$. Estos puntos se encuentran sobre la superficie de una esfera imaginaria cuyo centro, por conveniencia, puede coincidir con el punto de alimentación de la antena. El radio de la esfera debe ser igual o mayor a la distancia mínima que define al espacio donde inicia el campo de radiación. En la figura 4 se muestra el sistema de referencia de ejes coordenados para una antena arbitraria y uno de los puntos $\mathrm{P}$ de observación.

Si se considera el caso más general de una antena cuya geometría involucra corrientes con componentes en las direcciones $x, y, z$, las tres componentes del vector de potencial magnético $\vec{A}$ producido en un punto de observación $\mathrm{P}$ se pueden calcular como

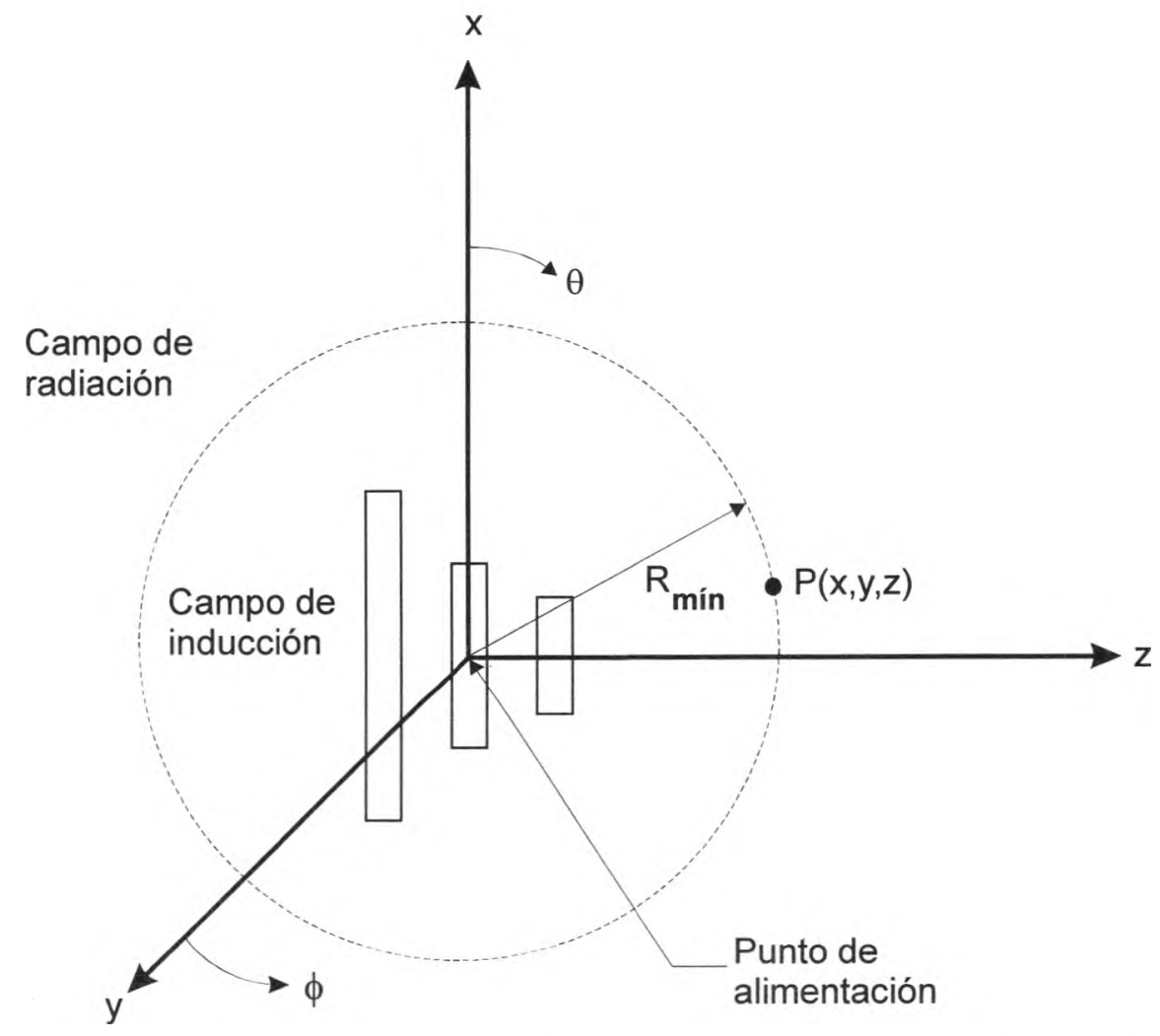

Figura 4. Sistema de ejes coordenados para calcular el patrón de radiación de la antena 


$$
A_{z}(P)=\frac{\mu}{4 \pi} \sum_{n=1}^{N} I_{n_{z}} \int_{\Delta l_{n}} \frac{e^{-j k r_{n}}}{r_{n}} d z^{\prime}
$$

donde $\mu$ es la permeabilidad del espacio libre en el aire $\left(\mu=\mu_{0}\right), \mathrm{N}$ es el número total de segmentos en la antena, $k$ es la constante de propagación, y $r_{n}$ es la distancia entre un punto fuente sobre el eje del segmento $n$ y el punto de observación P. Las variables de integración se denotan como $x^{\prime}, y^{\prime}, z^{\prime}$ para evitar confusión con los ejes " $x$ ", " $y$ ", " $z$ ", ya que los componentes de las corrientes que fluyen por los ejes de los conductores de la antena no son necesariamente colineales con los ejes del sistema de coordenadas, especialmente si hay varios tubos que la conforman.

Habiendo obtenido los tres componentes del vector $\vec{A}$ se puede determinar el campo magnético producido en el mismo punto de observación como

$$
\vec{H}=\frac{1}{\mu} \vec{\nabla} \times \vec{A}
$$

y finalmente, el campo eléctrico se obtiene como

$$
\vec{E}=\frac{1}{j \omega \varepsilon} \vec{\nabla} \times \vec{H}
$$

La magnitud de este campo eléctrico se calcula a partir de los cuadrados de las partes reales e imaginarias de sus tres componentes, " $x$ ", " $y$ ", " $z$ ".

$$
\begin{aligned}
\left|E_{T}\right|= & {\left[\operatorname{Re}\left(E_{x}\right)^{2}+\operatorname{Re}\left(E_{y}\right)^{2}+\operatorname{Re}\left(E_{z}\right)^{2}+\right.} \\
& \left.\operatorname{Im}\left(E_{x}\right)^{2}+\operatorname{Im}\left(E_{y}\right)^{2}+\operatorname{Im}\left(E_{z}\right)^{2}\right]^{\frac{1}{2}}
\end{aligned}
$$

De esta manera, variando los ángulos $\theta$ y $\phi$ de la figura 4 , se puede calcular la intensidad del campo eléctrico en puntos $\mathrm{P}$ de observación suficientes para graficar los patrones de radiación en cualquier plano.

\section{2) Ancho del haz principal}

En la dirección de máxima radiación, el ancho del haz, $\alpha$, se define como se indica en la figura 5 . Es el ángulo formado entre las rectas que pasan por el origen y los puntos donde la intensidad del campo eléctrico es igual a $0.707 \mathrm{E}_{\text {máximo }}$. Nótese que, como la radiación puede ser diferente en los planos $\mathrm{E}$ y $\mathrm{H}$, los ángulos correspondientes que definen al ancho del haz son diferentes en cada caso.
3) Relación de intensidad del campo hacia el frente y hacia atrás

Basándose en las figuras 4 y 5 , esta relación se define como:

$$
R=\frac{E\left(\theta=90^{\circ}, \phi=90^{\circ}\right)}{E\left(\theta=90^{\circ}, \phi=270^{\circ}\right)}
$$

suponiendo que la máxima radiación ocurre en la dirección positiva de $z$.

\section{4) Impedancia de entrada}

La impedancia de entrada se obtiene a partir de la matriz de admitancias $[Y]$, es decir, de $[Z]^{-1}$, que contiene toda la información de todos los acoplamientos entre el punto de alimentación y el resto de la antena:

$$
Z_{\text {entrada }}=\frac{1}{Y_{i, i}}
$$

donde $i$ es el número correspondiente al segmento donde se alimenta la antena.

\section{5) Ganancia}

Por definición, la ganancia de una antena A es igual a

$$
\mathrm{G}=\frac{\text { Densidad de potencia máxima producida por la antena } A}{\text { Densidad de potencia promedio de una antena de referencia }}
$$

Si se conoce la intensidad del campo eléctrico en la dirección de máxima radiación, $E_{\text {máx }}$, el numerador de la relación anterior se calcula como

$$
P_{\text {máx }}=\frac{E_{\text {máx }}^{2}}{2 Z_{0}}
$$

donde $Z_{0}$ es la impedancia característica del espacio libre e igual a 377 o $120 \pi \Omega$.

El denominador de la misma relación se obtiene en función de la corriente en el punto de alimentación, $\mathrm{I}_{0}$, la parte real de la impedancia de entrada, $R_{\text {entrada }}$, y el radio $R$ de la esfera imaginaria sobre la cual se calculó la intensidad del campo eléctrico:

$$
P_{\text {prom }}=\left(\frac{1}{2} I_{0}^{2} R_{\text {ent }}\right) / 4 \pi R^{2}
$$




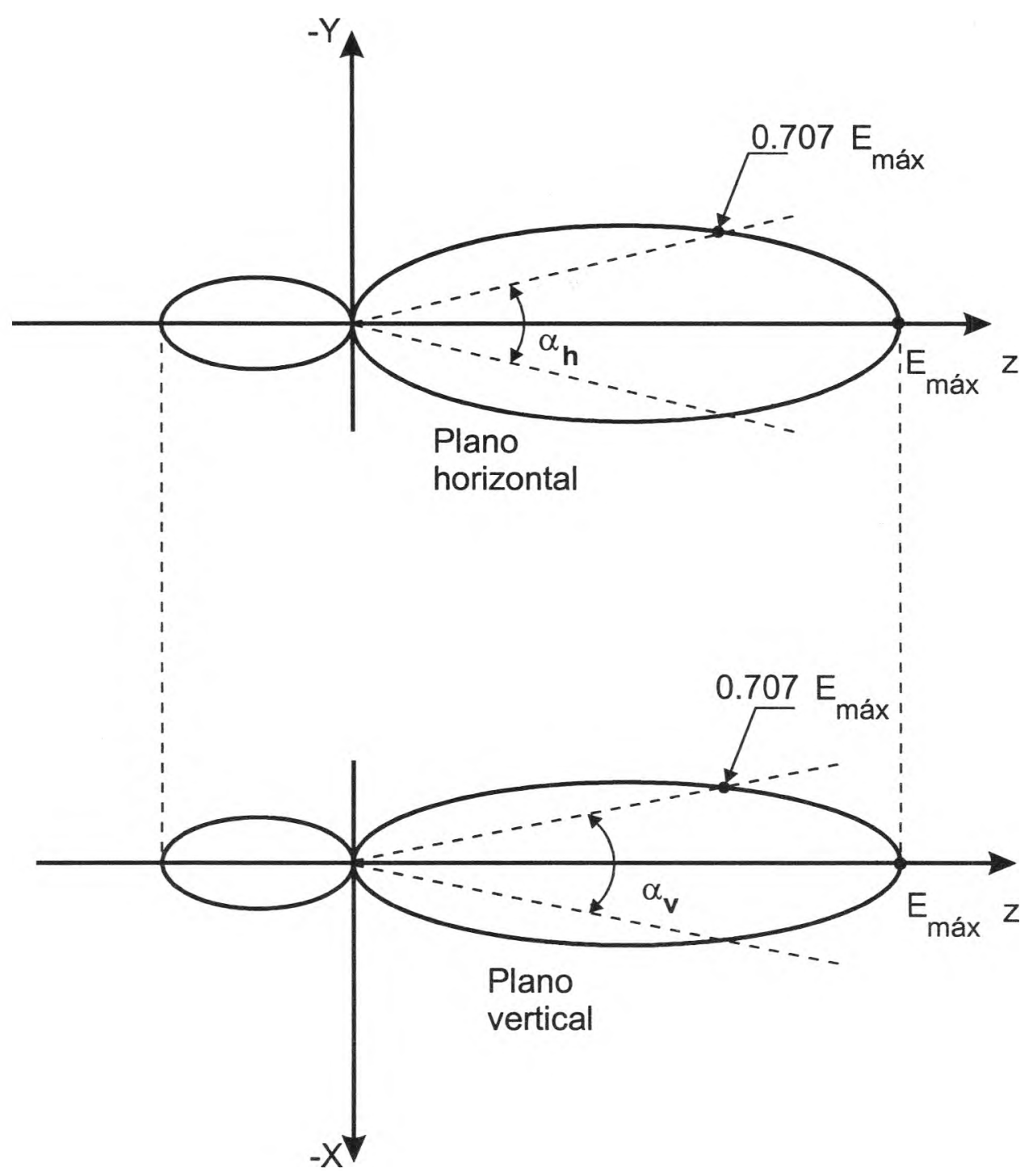

Figura 5. Definición del ancho del haz de radiación principal

Por lo tanto, sustituyendo las ecuaciones 24 y 25 en la relación original, se tiene que:

$$
\text { Ganancia }=\frac{4 \pi R^{2} E_{\text {máx }}^{2}}{Z_{0} I_{0}^{2} R_{e n t}}
$$

\section{Ejemplos de algunas distribuciones de corrientes}

En las figuras 6 y 7 se muestran las corrientes escalonadas obtenidas para un dipolo de longitud dividido, en cinco y nueve segmentos respectivamente. Se observa que al pasar de $\mathrm{N}=5$ a $\mathrm{N}=9$, la aproximación de la corriente se acerca más a la distribución senoidal. En ambas figuras, la corriente vale cero en los extremos de los medios segmentos que se deben considerar al final de cada tubo (compárese la figura 2 con la 6).

Pasando a una antena más compleja, en la figura 8 se muestra la distribución obtenida en los cinco elementos de una antena Yagi ( Ortiz, 1998). La longitud total de cada elemento y la separación entre ellos se indica en la misma figura, así como la posición del dipolo alimentado (es decir, $V_{18}=1 \angle 0^{\circ}$ volts y los demás $V$ son iguales a cero). En este caso, por simplicidad y reducción de espacio, sólo se muestra la magnitud de la corriente en cada segmento de los cuatro elementos parásitos y del elemento alimentado.

El lector interesado en obtener más información sobre la aplicación del método y cálculos de patrones de radiación, ganancia, etc., para antenas más complejas con elementos curvos, puede consultar otras publicaciones (Neri, 1979,1980). 


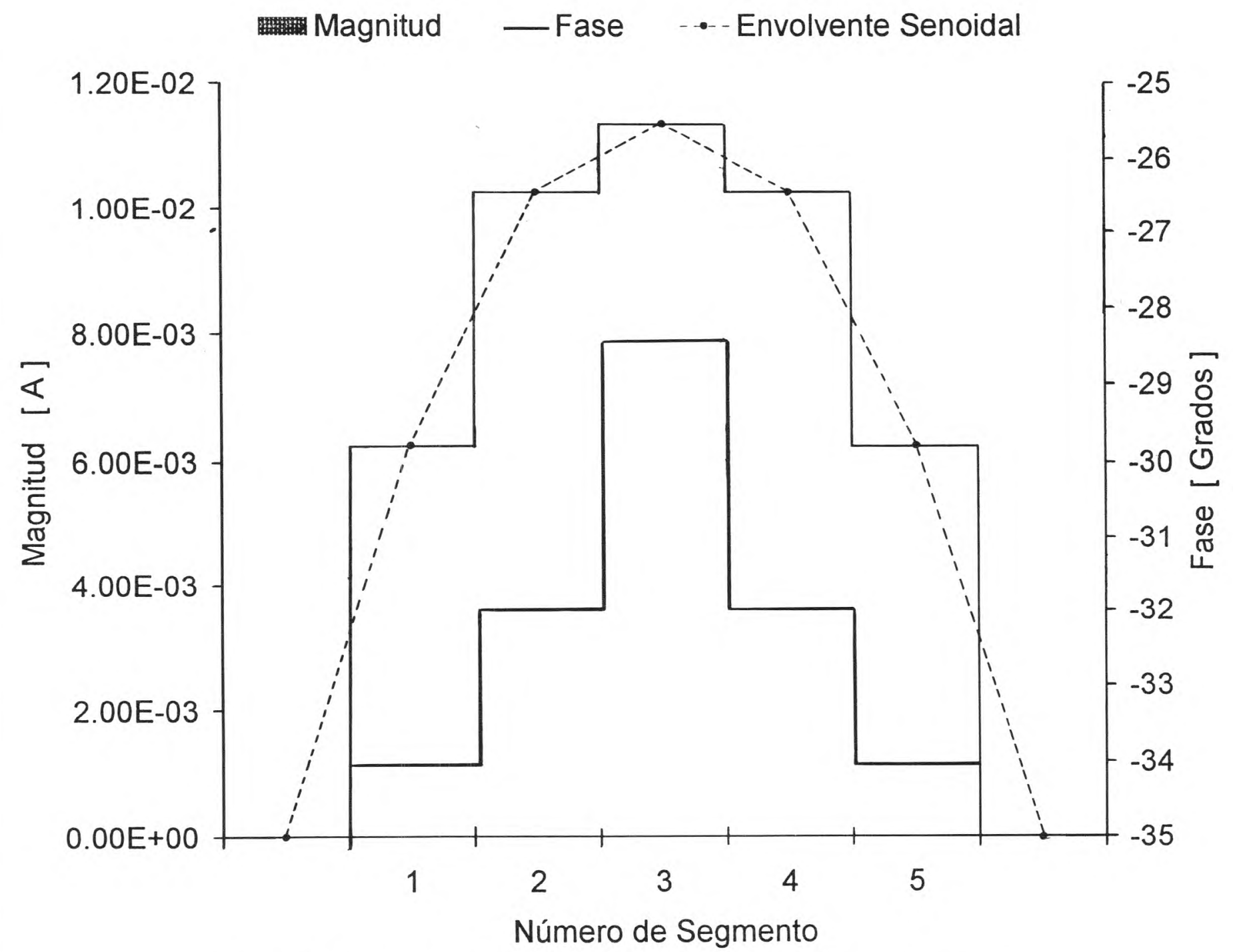

Figura 6. Distribución de corrientes para un dipolo de $\lambda / 2$ con 5 segmentos

\section{Conclusiones}

Se ha presentado un procedimiento matemático sencillo y preciso del método de momentos para analizar las propiedades de operación de un gran número de antenas empleadas en radiocomunicaciones. El procedimiento emplea funciones escalón para aproximar o discretizar la distribución de las corrientes en los conductores. El procedimiento es de actualidad e importancia tanto académica como práctica y constituye una herramienta didáctica que complementa la literatura existente, de modo que puede ser útil para alumnos y profesores de ingeniería en licenciatura y posgrado, fabricantes y profesionales.

\section{Referencias}

Harrington R.F. (1968). Field Computation by Moment Methods. Macmillan, Nueva York.

Tsai L.L. y Smith Ch. E. (1978). Moment Methods in Electromagnetics for Undergraduates. IEEE Transactions on Education, Vol. E-21, No. 1, 14-22.

Neri R. y MacLean T.S.M. (1978). Axial-line-current Approximation for Short Cylindrical Wires. Electronics Letters, Vol. 14, No. 24, 776-778.

Neri R. y MacLean T.S.M. (1979). Synthesis of a Small Parabolic Grid Receiving Antenna. IEEE Transactions on Antennas and Propagation, Vol.27, No. 4, 524-527. 
Neri R. (1980). Self and Mutual Impedances Between Sub-elements of Dipole Antennas: Contributions from Currents and Charges. Int. J. Electronics, Vol. 48, No. 5, 435-442.

Neri R. (1980). Power gain of Cassegrain Antennas with Small Gridded Hyperboloidal Sub-reflectors. IEE Proceedings, Pt. H., Vol. 127, No. 5.

Wang J.J.H. (1990). Generalised Moment Methods in Electromagnetics. IEE Proceedings, Vol. 137, Pt. H., No. 2, 127-132.

Ortiz J. (1998). Diseño de una antena de UHF para recepción de TV de alta definición por el método de momentos. Tesis profesional, Universidad Nacional Autónoma de México, Facultad de Ingeniería, Ciudad Universitaria, México.

\section{Agradecimientos}

El autor agradece a Omar Gabriel Torres Gómez y Saul Lazcano Salas su colaboración en la captura de los textos y figuras de este artículo, así como a Josué Ortiz Ramírez por programar y obtener los resultados mostrados en las figuras 6,7 y 8 .

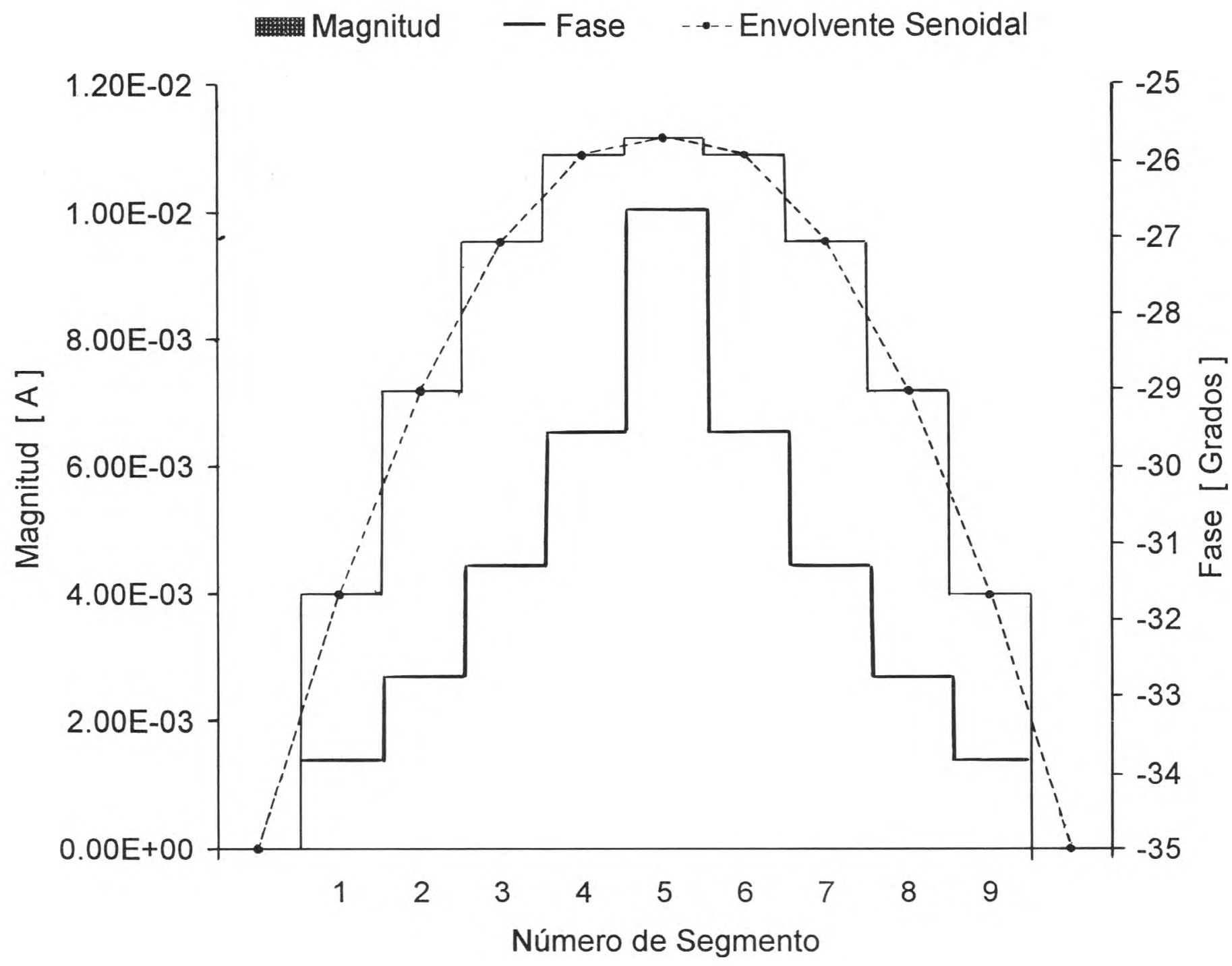

Figura 7. Distribución de corrientes para un dipolo de $\lambda / 2$ con 9 segmentos 

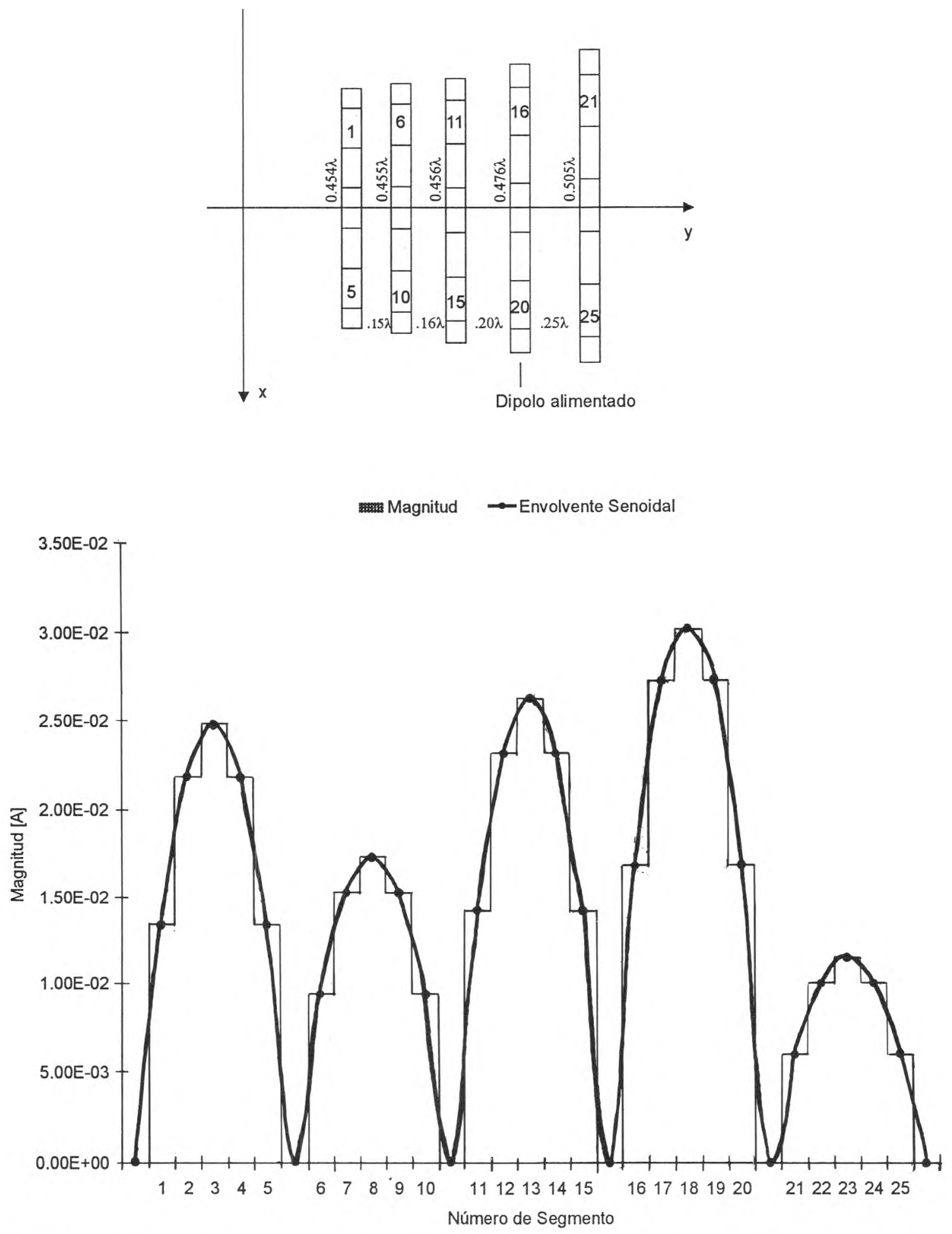

Figura 8. Distribución de corrientes para una antena Yagi de 5 elementos (total: 25 segmentos)

\section{Semblanza del autor}

Rodolfo Neri-Vela. Nació el 19 de febrero de 1952 en Chilpancingo, Gro. Cursó la carrera de ingeniero mecánico electricista, con especialidad en comunicaciones y electrónica, en la Facultad de Ingeniería de la Universidad Nacional Autónoma de México (UNAM); en 1976 cursó su maestría en telecomunicaciones en la Universidad de Essex, Inglaterra, becado por el Consejo Británico, y tres años después obtuvo el grado de doctor en radiación electromagnética aplicada por la Universidad de Birmingham, Inglaterra, como becario del Consejo Nacional de Ciencia y Tecnología. Durante 19 años ha sido profesor de diversas asignaturas en la Facultad de Ingeniería de la UNAM, donde actualmente labora en el Departamento de Telecomunicaciones. 